\title{
Analysis of the causal behavior in energy transfer between atoms
}

\author{
E. A. Power and T. Thirunamachandran \\ Department of Mathematics and Department of Chemistry, University College London, London WC1E 6BT, England
}

(Received 21 April 1997)

\begin{abstract}
A critical analysis of the problem of causality in the energy transfer between a pair of atoms is carried out. One of the pair, the source, is initially excited and the other, the detector, is in its ground state. The probabilities are calculated for the detector to be excited at a later time $t$ under different experimental conditions. In the first, a precise specification is made of the state of the source and that of the radiation field at the observation time $t$. In the second, the final state of the field is unspecified, whereas in the third, the state of neither the field nor the source is specified at time $t$. These three cases correspond to predicting the outcomes of different experimental measurements. It is shown that of the three cases, only the third exhibits exact causal behavior. The other two are noncausal. The noncausal contributions to the probability arise from the correlation of vacuum fluctuations at the two atoms at different times in the interval 0 to $t$. The probabilities for these cases can be significant for times slightly less than $R / c$, where $R$ is the interatomic separation. Explicit expressions, correct to fourth order in the transition moments, for the probabilities in the three cases are given. A proof of strict causality to all orders is presented for the third case where the final measurement is solely made on the detector. In Appendix A the related problem of the time dependence of the electric energy density associated with the source is analyzed and shown to be causal. [S1050-2947(97)03410-0]

PACS number(s): 03.65.Bz, 32.80.-t, 42.50.-p
\end{abstract}

\section{INTRODUCTION}

The energy transfer between a pair of atoms and the role of causality is a long-standing problem in the quantum theory of atom-radiation interaction. This was first studied using perturbation theory by Fermi [1] in connection with the propagation of light in vacuum. An earlier paper by Kikuchi [2], often referred to in this context, however, addresses the causality question in relation to the emission of radiation from a single atom by considering the time development of the field energy density in its neighborhood. In these and later papers [3-5], and in textbooks, e.g., Louisell [6], various approximations were employed that led to causal behavior. They include the neglect of terms with nonresonant denominators in the probability amplitudes and the extension of the integrals that appear in these amplitudes to the negative-frequency domain. It was pointed out by Shirokov [7] and by others [8-10] that a calculation without these approximations gave a noncausal result for the probability of energy transfer. In terms of the interatomic separation $R$, the expected causal result would be that the probability $P(t)$ vanishes exactly for $t<R / c$. Shirokov found that the noncausal component of the probability can be large in the interval $(R-\lambda) / c<t<(R+\lambda) / c$, where $\lambda$ is the reduced wavelength for a typical atomic transition. Rubin [9] calculated the probability amplitude to lowest order in the transition moments using time-dependent perturbation theory and extracted from it the noncausal term. The corresponding probability for $t<R / c$ was examined in the interval $0<t$ $<(R-\lambda) / c$, outside the Shirokov domain, and found to be very small. In a later paper Craig and Thirunamachandran [10] confirmed the probability to be noncausal and obtained an explicit expression for it in terms of ci and si functions. They suggested that the noncausal behavior may be due to the limits imposed by the time-energy uncertainty relation- ship on the specification of energy eigenstates at a given time.

Ferretti [11], Valentini [8], and Craig and Thirunamachandran [10] pointed out that an examination of the problem within a wider framework could restore causality. In the earlier works the time-dependent probability was calculated for a complete specification of the final state. In addition to the absorber being excited, the emitter was specified to be in its ground state and the field was in the vacuum state at time $t$. Clearly, alternative questions that are closer to realistic experimental situations can be posed. For example, one could ask for the inclusive probability of finding the absorber excited, without making observations on either the emitter or the field. In Sec. II we frame three questions involving sharply defined conditions. The first of these relates to the historic Fermi problem and the last refers to the aforementioned inclusive question. The second concerns an intermediate situation with a less stringent specification compared to the first. It is interesting to find in the literature statements that mirror all these three cases. The question dealing with the Fermi problem is clearly stated by Louisell [6]: "The problem is to find the probability that, at time $t$, the final state in which the emitter atom has decayed and the photon has been absorbed by the receiver atom is given by |(receiver) excited, (emitter) ground; (radiation) vacuum $\rangle$." 'The second case where no specification is made of the final state of the radiation field has been enunciated by Shirokov [7(a)]: "At $t=0$ one atom is excited and the other atom is in the ground state. Initially there are no photons. We compute the probability of finding the second atom in the excited state at time $t$, the first atom being in the ground state." The third case in which the final specification is that for the receiver atom alone has been raised by Craig and Thirunamachandran [10]: "We should calculate the probability of the receiver atom being excited at time $t$ without making any reference to the [final states of the] emitter atom and the field.' This is 
also implicit in calculations that focus on the time evolution of the occupation operator as in the work of Compagno and co-workers [12] and Milonni, James, and Fearn [13]. Models used in discussions of photodetection [14] also ask questions about the state of the detector alone; in this respect they resemble the third case. In this context it is of interest to note Ferretti's early remarks [11] that the "troubles have their origin in the fact that if a velocity is to be correctly and rigorously defined we have to use information concerning only objects that can be rigorously localized in space and time; in our case the information must only be related to that of the detector. Instead, in Fermi's case, other information is required concerning the number of quanta, that is, the constraint that in the final state that number is zero." In a very recent paper Kaup and Rupasov [15] claim to have reexamined the Fermi problem and to have found no violation of causality. However, their calculations were based on a model described by an effective Hamiltonian and not on the real physical system. In fact, their calculation has causality built in by virtue of their extension to negative frequencies of the relevant integrals.

In the papers referred to above, the initial and final states of atoms were taken to be bare states. Attempts $[7(b), 16]$ to go further in terms of dressed atoms (renormalized states) are fraught with difficulty. The dressed states of a pair of atoms by their very nature involve the interatomic separation $R$. Hence the initial specification is necessarily nonlocal and it is not possible to formulate sharp questions of causality [17]. For causal questions to be meaningful it is essential to have local specification of the initial state. For example, it is meaningful to ask whether the time evolution of the electromagnetic fields and their energy densities arising from a single excited atom are causal. This in fact was the question first studied by Kikuchi [2] in 1930 and the problem of detection did not arise. He used time-dependent states perturbed to first order to obtain an expression for the electric energy density quadratic in the transition moments. He found the propagation to be causal after extending the relevant integrals to negative frequencies. We show in Appendix A that to obtain a complete expression for the energy density correct to second order it is necessary to employ states perturbed to second order in the moments. In this calculation the additional terms provide the precise contribution needed to extend the integrals to the negative-frequency domain. Thus the time evolution of the electromagnetic energy densities is strictly causal.

In Fermi's treatment [1] of the propagation of light, the dynamics of the detector atom was taken into account and the problem became one of energy transfer between atoms. In the present work we study this problem with special reference to the three cases referred to above. In Sec. II the probabilities for the three cases are formally expressed and in Sec. III the methodology to find expressions for these probabilities is outlined. The equations of motion for the creation and annihilation operators for both photons and electrons are found from the quantum electrodynamical Hamiltonian for a pair of atoms interacting with the radiation field. From these coupled equations, the Heisenberg operator for the Maxwell field $\vec{d}(\vec{r}, t)$ is obtained as the sum of the source-free and source-dependent fields, the latter being fully retarded. The retarded nature of the source terms is used in Sec. IV to give a formal proof, to all orders in the dipole moments, that the predicted probability $P_{\mathrm{III}}(t)$ for case III is exactly zero for $t<R / c$. In Sec. V the explicit expressions for the probability in all three cases are found to fourth order. The probability $P_{\mathrm{I}}(t)$ for the first case is not causal, confirming the previous calculations $[7(\mathrm{a}), 9,10]$. The probability $P_{\mathrm{II}}(t)$ for case II contains noncausal terms in addition to those in $P_{\mathrm{I}}(t)$. However, for case III, both of these noncausal terms are found to cancel terms arising from interference of first- and thirdorder probability amplitudes. This provides explicit confirmation to fourth order of the general result given in Sec. IV.

\section{STATEMENT OF THE PROBLEM}

We consider the specific problem of excitation transfer between two atoms: $S$ the source and $D$ the detector. The atoms are taken to be fixed at $\vec{R}_{S}$ and $\vec{R}_{D}$, with $S$ in the excited state $|p\rangle$ and $D$ in its ground state $|g\rangle$ at the initial time $t=0$ and the Maxwell field is assumed to be in its ground state, namely, the vacuum $|\mathrm{vac}\rangle$. With the notation $|D, S ; F\rangle$ for a state of the composite system, the initial state is $|g, p ; \mathrm{vac}\rangle$. The well-known question considered by Fermi is the following: Given that at $t=0$, the state of the system was $|g, p ; \mathrm{vac}\rangle$, what is the probability of finding the system with detector atom $D$ in the excited state $|q\rangle$, source atom $S$ in its ground state $|g\rangle$ and the field in its vacuum state at a later time $t$ ? To answer this question experimentally one would require simultaneous measurements to be made on $D$, $S$, and the field at $t$. This of course is experimentally demanding. Other questions of a similar nature may also be asked. For example, given the same initial conditions, what is the probability of finding $D$ in the excited state $|q\rangle$ and $S$ in its ground state $|g\rangle$ at time $t$ ? Here the final state of the field is not specified. A question much more amenable to experiment is the following: Given the same initial conditions, what is the probability of finding the atom $D$ in the excited state $|q\rangle$ at time $t$ ? The relevant experiment to answer this question is less demanding since only a measurement on $D$ is needed at time $t$. No final specification of the states of $S$ and of the field is made.

In Secs. III and V we consider these three cases and calculate the probabilities of excitation transfer using the theory of nonrelativistic quantum electrodynamics. The first, case I, is the original Fermi problem. Case II deals with the intermediate question where the field is unspecified at $t$ and case III with the one-center measurement question, namely, $D$ alone is specified at time $t$. The initial state for each of the three cases is

$$
|i\rangle=|g, p ; \mathrm{vac}\rangle .
$$

The corresponding probabilities $P(t)$ are: case I,

$$
P_{\mathrm{I}}(t)=\left|\left\langle f\left|e^{i H_{0} t / \hbar} e^{-i H t / \hbar}\right| i\right\rangle\right|^{2},
$$

with $|f\rangle$ in our notation given by

$$
|f\rangle=|q, g ; \mathrm{vac}\rangle \text {; }
$$

case II, 


$$
P_{\mathrm{II}}(t)=\sum_{F}\left|\left\langle F ; g, q\left|e^{i H_{0} t / \hbar} e^{-i H t / \hbar}\right| i\right\rangle\right|^{2},
$$

where $|F\rangle$ is a complete set of field states including the vacuum; and case III,

$$
P_{\mathrm{III}}(t)=\sum_{F} \sum_{S}\left|\left\langle F ; S, q\left|e^{i H_{0} t / \hbar} e^{-i H t / \hbar}\right| i\right\rangle\right|^{2},
$$

where $|S\rangle$ is a complete set of atomic states of $S$.

\section{METHODOLOGY}

For the processes under discussion, the dynamics is governed by the multipolar Hamiltonian (3.1) in secondquantized form

$$
H=H_{\text {atoms }}+H_{\text {rad }}+H_{\text {int }},
$$

where

$$
\begin{gathered}
H_{\text {atoms }}=\sum_{n} E_{n}^{D} b_{n}^{\dagger D} b_{n}^{D}+\sum_{n} E_{n}^{S} b_{n}^{\dagger S} b_{n}^{S}, \\
H_{\text {rad }}=\sum_{\text {modes }} \hbar \omega a^{\dagger} a .
\end{gathered}
$$

In the electric-dipole approximation

$$
\begin{aligned}
H_{\mathrm{int}}= & -\sum_{m, n} \vec{\mu}^{m n}(D) b_{m}^{\dagger D} b_{n}^{D} \cdot \vec{d}\left(\vec{R}_{D}\right) \\
& -\sum_{m, n} \vec{\mu}^{m n}(S) b_{m}^{\dagger S} b_{n}^{S} \cdot \vec{d}\left(\vec{R}_{S}\right) .
\end{aligned}
$$

In Eqs. (3.2)-(3.4) $b$ and $b^{\dagger}$ are the annihilation and creation operators for atomic states and $a$ and $a^{\dagger}$ are the annihilation and creation operators for photons. These obey the standard anticommutation-commutation relations. The displacement vector field operator $\vec{d}(\vec{r})$ in Eq. (3.4) is given by the mode expansion

$$
\vec{d}(\vec{r})=i \sum_{\text {modes }}\left(\frac{2 \pi \hbar c k}{V}\right)^{1 / 2}\left(\vec{e} a e^{i \vec{k} \cdot \vec{r}}-\overrightarrow{\bar{e} a} a^{\dagger} e^{-i \vec{k} \cdot \vec{r}}\right) .
$$

The equations of motion for the operators $a$ and $b_{n}$ are

$$
\begin{aligned}
\dot{a}= & \frac{1}{i \hbar}[a, H]=-i \omega a+\frac{1}{\hbar}\left(\frac{2 \pi \hbar c k}{V}\right)^{1 / 2} \\
& \times \sum_{m, n} \vec{\mu}^{m n}(D) \cdot \overrightarrow{\bar{e} e}-i \vec{k} \cdot \vec{R}_{D} b_{m}^{\dagger D} b_{n}^{D}+\frac{1}{\hbar}\left(\frac{2 \pi \hbar c k}{V}\right)^{1 / 2} \\
& \times \sum_{m, n} \vec{\mu}^{m n}(S) \cdot \overrightarrow{\bar{e} e}-i \vec{k} \cdot \vec{R}_{S} b_{m}^{\dagger S} b_{n}^{S}
\end{aligned}
$$

and

$$
\dot{b}_{n}=\frac{1}{i \hbar}\left[b_{n}, H\right]=-\frac{i}{\hbar} E_{n} b_{n}+\frac{i}{\hbar} \sum_{m} \vec{\mu}^{m n} \cdot \vec{d}(\vec{R}) b_{m} .
$$

The atomic label is implicit in Eq. (3.7). The simple form of Eq. (3.7), i.e., its linearity in the $b$ 's, is a direct consequence of the use of the multipolar formalism.

We now express the amplitudes that appear in the probabilities (2.2), (2.4), and (2.5) for the three cases in the Heisenberg picture. For case I, where the final state is $|q, g ; \mathrm{vac}\rangle$, the amplitude is

$$
\begin{aligned}
& \left\langle\mathrm{vac}, g, q\left|e^{i H_{0} t / \hbar} e^{-i H t / \hbar}\right| g, p ; \mathrm{vac}\right\rangle \\
& \quad=\left\langle\operatorname{vac} ; \overline{0}\left|b_{g}^{S}(0) b_{q}^{D}(0) e^{-i H t / \hbar}\right| g, p ; \mathrm{vac}\right\rangle e^{i E_{q}^{D} t / \hbar} e^{i E_{g}^{S} t / \hbar} \\
& =\left\langle\operatorname{vac} ; \overline{0}\left|e^{-i H t / \hbar} b_{g}^{S}(t) b_{q}^{D}(t)\right| g, p ; \operatorname{vac}\right\rangle e^{i E_{q}^{D} t / \hbar} e^{i E_{g}^{S} t / \hbar},
\end{aligned}
$$

where the Heisenberg operators are given by

$$
b_{n}(t)=e^{i H t / \hbar} b_{n}(0) e^{-i H t / \hbar} .
$$

The state $|\overline{0}\rangle$ in Eq. (3.8) refers to the no-particle fermion state with the properties $b_{n} \mid \overline{0\rangle}=0$ and $b_{n}^{\dagger}|\overline{0}\rangle=|n\rangle$. The state $\mid \overline{0}$; vac $\rangle$ is the vacuum state of the composite system satisfying

$$
H|\overline{0} ; \mathrm{vac}\rangle=0
$$

Using the interaction representation $b_{n}(t)=\exp \left[-i E_{n} t /\right.$ $\hbar] \beta_{n}(t)$ and Eq. (3.10), the amplitude (3.8) for case I becomes

$$
\left\langle\operatorname{vac} ; \overline{0}\left|\beta_{g}^{S}(t) \beta_{q}^{D}(t)\right| g, p ; \mathrm{vac}\right\rangle .
$$

Hence the probability of finding the detector in state $|q\rangle$, the source atom $S$ in state $|g\rangle$, and the field in its vacuum state at time $t$ is

$$
P_{\mathrm{I}}(t)=\left|\left\langle\operatorname{vac} ; \overline{0}\left|\beta_{g}^{S}(t) \beta_{q}^{D}(t)\right| g, p ; \mathrm{vac}\right\rangle\right|^{2} .
$$

An evaluation of this probability to fourth order is given in Sec. V.

For case II, where the field is unspecified, two-photon amplitudes such as

$$
\begin{aligned}
& \left\langle k^{\prime}, k ; g, q\left|e^{i H_{0} t / \hbar} e^{-i H t / \hbar}\right| g, p ; \mathrm{vac}\right\rangle \\
& =\left\langle\operatorname{vac}, \overline{0} \bar{\alpha} \alpha_{k}(t) \alpha_{k^{\prime}}(t) \beta_{g}^{S}(t) \beta_{q}^{D}(t) \mid g, p ; \mathrm{vac}\right\rangle
\end{aligned}
$$

contribute in addition to Eq. (3.11). In Eq. (3.14) we have used the interaction representation for the field operators $a(t)=\exp [-i \omega t] \alpha(t)$. The probability that $D$ is in state $|q\rangle$ and $S$ in $|g\rangle$ at time $t$ is

$$
P_{\mathrm{II}}(t)=\sum_{F}\left\langle i\left|\beta_{q}^{D^{\dagger}}(t) \beta_{g}^{S \dagger}(t)\right| \overline{0} ; F\right\rangle\left\langle F ; \overline{0}\left|\beta_{g}^{S}(t) \beta_{q}^{D}(t)\right| i\right\rangle,
$$

where the sum is over a complete set of field states. Closure over field states gives

$$
P_{\mathrm{II}}(t)=\left\langle i\left|\beta_{q}^{D^{\dagger}}(t) \beta_{g}^{S^{\dagger}}(t) \beta_{g}^{S}(t) \beta_{q}^{D}(t)\right| i\right\rangle,
$$

which is evaluated in Sec. V. 
When the state of $S$ is also unspecified at the time of measurement (case III), the probability of finding the detector in state $|q\rangle$ is

$$
\begin{aligned}
P_{\mathrm{III}}(t) & =\sum_{p^{\prime}, F}\left\langle i\left|\beta_{q}^{D^{\dagger}}(t) \beta_{p^{\prime}}^{S \dagger}(t)\right| \overline{0 ;} F\right\rangle\left\langle F ; \overline{0 \mid} \beta_{p^{\prime}}^{S}(t) \beta_{q}^{D}(t) \mid i\right\rangle \\
& =\left\langle i\left|\beta_{q}^{D^{\dagger}}(t) \beta_{q}^{D}(t)\right| i\right\rangle,
\end{aligned}
$$

where we have used closure over the source states in addition to those over the field. A typical amplitude contributing to the probability (3.17) is

$$
\left\langle\operatorname{vac} ; \overline{0}\left|\alpha_{k}(t) \beta_{p^{\prime}}^{S}(t) \beta_{q}^{D}(t)\right| g, p ; \mathrm{vac}\right\rangle
$$

The amplitudes and probabilities are calculated using perturbation theory in Sec. V. The probabilities are evaluated to the order of $\mu^{2}(S) \mu^{2}(D)$ and the $R$-dependent terms extracted.

For the calculations in Secs. IV and V we need the Heisenberg equations for the $\alpha$ and $\beta$ operators. The equation of motion for the annihilation operator $\beta_{n}(t)$ for electron states is, from Eq. (3.7),

$$
\dot{\beta}_{n}(t)=\frac{i}{\hbar} \sum_{m} \vec{\mu}^{m n} \cdot \vec{d}(\vec{R}, t) \beta_{m}(t) e^{-i \omega_{m n} t},
$$

from which

$$
\beta_{n}(t)=\beta_{n}(0)+\frac{i}{\hbar} \sum_{m} \int_{0}^{t} d t^{\prime} \vec{\mu}^{m n} \cdot \vec{d}\left(\vec{R}, t^{\prime}\right) \beta_{m}\left(t^{\prime}\right) e^{-i \omega_{m n} t^{\prime}} .
$$

The total displacement vector field $\vec{d}\left(\vec{r}, t^{\prime}\right)$ in the integrand of Eq. (3.20) is evaluated at $\vec{R}_{S}$ for $\beta_{n}^{s}(t)$ and at $\vec{R}_{D}$ for $\beta_{n}^{D}(t)$.

Similarly, for the $\alpha$ operators, we have from Eq. (3.6)

$$
\begin{aligned}
& \dot{\alpha}(t)=\frac{1}{\hbar}\left(\frac{2 \pi \hbar c k}{V}\right)^{1 / 2} \sum_{m, n} \vec{\mu}^{m n}(S) \cdot \vec{e} e^{-i \vec{k} \cdot \vec{R}_{S}} e^{i \omega t}
\end{aligned}
$$

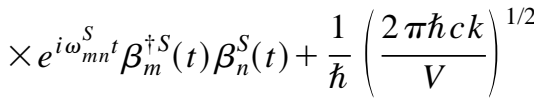

$$
\begin{aligned}
& \times \sum_{m, n} \vec{\mu}^{m n}(D) \cdot \vec{e} e^{-i \vec{k} \cdot \vec{R}_{D}} e^{i \omega t} \\
& \times e^{i \omega_{m n}^{D} t} \beta_{m}^{\dagger D}(t) \beta_{n}^{D}(t) .
\end{aligned}
$$

The operator $\alpha(t)$ is then a sum of the free field and atomdependent terms

$$
\alpha(t)=\alpha(0)+\alpha^{S(s)}(t)+\alpha^{D(s)}(t),
$$

where the atom-dependent $\alpha$ 's have the form

$$
\begin{aligned}
\alpha^{(s)}(t)= & \frac{1}{\hbar} \sum_{m, n} \vec{\mu}^{m n} \cdot \vec{e} e^{-i \vec{k} \cdot \vec{R}}\left(\frac{2 \pi \hbar c k}{V}\right)^{1 / 2} \\
& \times \int_{0}^{t} d t^{\prime} e^{i\left(\omega+\omega_{m n}\right) t^{\prime}} \beta_{m}^{\dagger}\left(t^{\prime}\right) \beta_{n}\left(t^{\prime}\right) .
\end{aligned}
$$

For a single source, the Heisenberg operator for the displacement field $\vec{d}(\vec{r}, t)$ is

$$
\vec{d}(\vec{r}, t)=\vec{d}^{(0)}(\vec{r}, t)+\vec{d}^{(s)}(\vec{r}, t),
$$

where $\vec{d}^{(s)}(\vec{r}, t)$ is found from the mode expansion (3.5) using Eq. (3.23) [18]. We have

$$
\begin{aligned}
d_{i}^{(s)}(\vec{r}, t)= & \frac{i}{\hbar} \sum_{m, n} \sum_{\text {modes }} \mu_{j}^{m n}\left(\frac{2 \pi \hbar c k}{V}\right) \\
& \times e_{i} \overline{e_{j}} e^{i \vec{k} \cdot(\vec{r}-\vec{R})} e^{-i \omega t} \\
& \times \int_{0}^{t} d t^{\prime} e^{i\left(\omega+\omega_{m n}\right) t^{\prime}} \beta_{m}^{\dagger}\left(t^{\prime}\right) \beta_{n}\left(t^{\prime}\right)+\text { H.c. }
\end{aligned}
$$

The polarization sum and the angular integration over the direction of $\vec{k}$ give

$$
\begin{aligned}
d_{i}^{(s)}(\vec{r}, t)= & \frac{i c}{\pi} \sum_{m, n} \mu_{j}^{m n}\left(-\nabla^{2} \delta_{i j}+\nabla_{i} \nabla_{j}\right) \\
& \times \frac{1}{|\vec{r}-\vec{R}|} \int_{0}^{t} d t^{\prime} \int_{0}^{\infty} d k(\sin k|\vec{r}-\vec{R}|) \\
& \times\left[e^{-i \omega\left(t-t^{\prime}\right)}-e^{i \omega\left(t-t^{\prime}\right)}\right] e^{i \omega_{m n} t^{\prime}} \beta_{m}^{\dagger}\left(t^{\prime}\right) \beta_{n}\left(t^{\prime}\right)
\end{aligned}
$$

for $\vec{r} \neq \vec{R}$. The $k$ integral in Eq. (3.26) is

$$
\begin{aligned}
& \int_{0}^{\infty} d k(\sin k|\vec{r}-\vec{R}|)\left[e^{-i \omega\left(t-t^{\prime}\right)}-e^{i \omega\left(t-t^{\prime}\right)}\right] \\
& \quad=\frac{1}{2 i} \int_{-\infty}^{\infty} d k\left[e^{i k|\vec{r}-\vec{R}|}-e^{-i k|\vec{r}-\vec{R}|}\right] e^{-i k c\left(t-t^{\prime}\right)} \\
& \quad=-i \pi\left[\delta\left(|\vec{r}-\vec{R}|-c\left(t-t^{\prime}\right)\right)-\delta\left(|\vec{r}-\vec{R}|+c\left(t-t^{\prime}\right)\right)\right] .
\end{aligned}
$$

Hence, for $t>|\vec{r}-\vec{R}| / c$,

$$
\begin{aligned}
d_{i}^{(s)}(\vec{r}, t)= & \sum_{m, n} \mu_{j}^{m n}\left(-\nabla^{2} \delta_{i j}+\nabla_{i} \nabla_{j}\right) \frac{e^{i \omega_{m n}(t-|\vec{r}-\vec{R}| / c)}}{|\vec{r}-\vec{R}|} \\
& \times \beta_{m}^{\dagger}(t-|\vec{r}-\vec{R}| / c) \beta_{n}(t-|\vec{r}-\vec{R}| / c)
\end{aligned}
$$

Of course, for $t<|\vec{r}-\vec{R}| / c, d_{i}^{(s)}(\vec{r}, t)$ is zero, reflecting the causal character of the source field.

For a many-atom system, such as the source plus detector in the present problem, the Maxwell field is formally additive. Using Eq. (3.22), we can write

$$
\vec{d}(\vec{r}, t)=\vec{d}^{(0)}(\vec{r}, t)+\vec{d}^{S}(\vec{r}, t)+\vec{d}^{D}(\vec{r}, t),
$$

with $\vec{d}^{S}(\vec{r}, t)$ and $\vec{d}^{D}(\vec{r}, t)$ given by Eq. (3.28). However, we emphasize that the atom-dependent term $\vec{d}^{S}(\vec{r}, t)$ is not identical to its value in the absence of the detector. Similarly, 
$\vec{d}^{D}(\vec{r}, t)$ is not identical to the corresponding expression for the isolated detector. This is a consequence of the fact that the $\beta$ operators in expression (3.28) for the field do not refer to the initial time $t=0$. At any time other than $t=0$, the $\beta$ 's operate on the composite space of the field and both atoms.

It is of interest to note that the mixing of the source and detector operators first occurs in the third term in a perturbation expansion in dipole moments; the first- and secondorder terms are strictly additive. The first-order term is found by replacing the $\beta$ 's in Eq. (3.28) by their values at $t=0$, and this leads to the familiar result

$$
\begin{aligned}
d_{i}^{(1)}(\vec{r}, t)= & \sum_{m, n} \mu_{j}^{m n}(S)\left(-\nabla^{2} \delta_{i j}+\nabla_{i} \nabla_{j}\right) \\
& \times \frac{e^{i \omega_{m n}^{S}\left(t-\left|\vec{r}-\vec{R}_{S}\right| / c\right)}}{\left|\vec{r}-\vec{R}_{S}\right|} \beta_{m}^{\dagger S}(0) \beta_{n}^{S}(0) \\
& +\sum_{m, n} \mu_{j}^{m n}(D)\left(-\nabla^{2} \delta_{i j}+\nabla_{i} \nabla_{j}\right) \\
& \times \frac{e^{i \omega_{m n}^{D}\left(t-\left|\vec{r}-\vec{R}_{D}\right| / c\right)}}{\left|\vec{r}-\vec{R}_{D}\right|} \beta_{m}^{\dagger D}(0) \beta_{n}^{D}(0) .
\end{aligned}
$$

The additivity in second order follows by approximating the $\beta$ 's in Eq. (3.28) to first order [19]. Higher-order approximations to the displacement vector field have contributions that are nonadditive in the sense that they are dependent on the transition moments of both the source and the detector. Finally, it must be stressed that, despite the general nonadditive nature of $\vec{d}(\vec{r}, t)$, the fields are nevertheless strictly additive for $t<R / c=\left|\vec{R}^{D}-\vec{R}^{S}\right| / c$; that is, in this time interval $\vec{d}^{S}(\vec{r}, t)$ depends only on $\mu(S)$ and $\vec{d}^{D}(\vec{r}, t)$ only on $\mu(D)$.

\section{PROOF OF STRICT CAUSALITY FOR CASE III TO ALL ORDERS IN PERTURBATION THEORY}

In this section we prove that $P_{\mathrm{III}}(t)[\mathrm{Eq} .(3.17)]$ is causal to all orders, i.e., the $R$-dependent part of $P_{\mathrm{III}}(t)$ vanishes for times less than $R / c$, where $\vec{R}=\vec{R}_{D}-\vec{R}_{S}$, the interatomic separation. The probability $P_{\mathrm{III}}(t)$ can be expressed as the expectation value of the projection operator $\gamma_{q q}^{D}(t)$ for the detector being found in state $|q\rangle$,

$$
P_{\mathrm{III}}(t)=\left\langle\mathrm{vac} ; p, g\left|\gamma_{q q}^{D}(t)\right| g, p ; \mathrm{vac}\right\rangle .
$$

In terms of the fermion operators $\beta$, the projection operator is

$$
\gamma_{q q}(t)=\beta_{q}^{\dagger}(t) \beta_{q}(t)
$$

which is a diagonal term of the general operator

$$
\gamma_{m n}(t)=\beta_{m}^{\dagger}(t) \beta_{n}(t)
$$

To find an integral equation for $\gamma_{m n}(t)$ we first write down its equation of motion using the expression (3.21) for the $\beta$ 's,

$$
\begin{aligned}
\dot{\gamma}_{m n}(t) & =\dot{\beta}_{m}^{\dagger}(t) \beta_{n}(t)+\beta_{m}^{\dagger}(t) \dot{\beta}_{n}(t) \\
& =-\frac{i}{\hbar} \vec{\mu}^{u v} \cdot \vec{d}(\vec{R}, t) \gamma_{r s}(t) T_{m n, u v}^{r s}(t),
\end{aligned}
$$

with

$$
T_{m n, u v}^{r s}(t)=\delta_{u}^{r} \delta_{n}^{s} \delta_{m v} e^{-i \omega_{u v} t}-\delta_{u}^{s} \delta_{m}^{r} \delta_{n v} e^{i \omega_{u v} t}
$$

$T_{m n, u v}^{r s}(t)$ is a $c$ number independent of $\vec{\mu}$ and $\vec{R}$. Hence

$$
\gamma_{m n}(t)=\gamma_{m n}(0)-\frac{i}{\hbar} \int_{0}^{t} d t^{\prime} \vec{\mu}^{u v} \cdot \vec{d}\left(\vec{R}, t^{\prime}\right) T_{m n, u v}^{r s}\left(t^{\prime}\right) \gamma_{r s}\left(t^{\prime}\right)
$$

We now show that for $t<R / c, \gamma_{m n}^{D}(t)$ is independent of $R$; the causal nature of the probability $P_{\mathrm{III}}(t)$ follows as a special case.

The general operator $\gamma_{m n}^{D}(t)$ for the detector is found by iterating Eq. (4.6):

$$
\begin{aligned}
\gamma_{m n}^{D}(t)= & \gamma_{m n}^{D}(0)-\frac{i}{\hbar} \int_{0}^{t} d t^{\prime}\left[\vec{\mu}^{u v}(D) \cdot \vec{d}\left(\vec{R}_{D}, t^{\prime}\right)\right] \\
& \times T_{m n, u v}^{r s}\left(t^{\prime}\right) \gamma_{r s}(0)+\left(-\frac{i}{\hbar}\right)^{2} \int_{0}^{t} d t^{\prime} \int_{0}^{t^{\prime}} d t^{\prime \prime} \\
& \times\left[\vec{\mu}^{u v}(D) \cdot \vec{d}\left(\vec{R}_{D}, t^{\prime}\right)\right]\left[\vec{\mu}^{u^{\prime} v^{\prime}}(D) \cdot \vec{d}\left(\vec{R}_{D}, t^{\prime \prime}\right)\right] \\
& \times T_{m n, u v}^{r s}\left(t^{\prime}\right) T_{r s, u^{\prime} v^{\prime}}^{r^{\prime} s^{\prime}}\left(t^{\prime \prime}\right) \gamma_{r^{\prime} s^{\prime}}^{D}(0)+\cdots,
\end{aligned}
$$

with the $N$ th term given by

$$
\begin{aligned}
& \left(-\frac{i}{\hbar}\right)^{N} \int_{0}^{t} d t_{1} \int_{0}^{t_{1}} d t_{2} \cdots \int_{0}^{t_{N-1}} d t_{N}\left[\vec{\mu}(D) \cdot \vec{d}\left(\vec{R}_{D}, t_{1}\right)\right] \\
& \times\left[\vec{\mu}(D) \cdot \vec{d}\left(\vec{R}_{D}, t_{2}\right)\right] \cdots\left[\vec{\mu}(D) \cdot \vec{d}\left(\vec{R}_{D}, t_{N}\right)\right] \\
& \times T\left(t_{1}\right) T\left(t_{2}\right) \cdots T\left(t_{N}\right) \gamma_{r_{N} s_{N}}^{D}(0),
\end{aligned}
$$

where the indices labeling the states of the detector are implicit. In Eq. (4.8) the displacement vector field is the total field evaluated at the detector. For $0 \leqslant t<R / c, t_{i}$ is also less than $R / c$ and $\vec{d}^{S}\left(\vec{R}_{D}, t_{i}\right) \equiv 0$, so that

$$
\vec{d}\left(\vec{R}_{D}, t_{i}\right)=\vec{d}^{(0)}\left(\vec{R}_{D}, t_{i}\right)+\vec{d}^{D}\left(\vec{R}_{D}, t_{i}\right) .
$$

Now $\vec{d}^{D}\left(\vec{R}_{D}, t_{i}\right)$ is independent of $\mu(S)$ and hence of $R$. Therefore, $\vec{d}\left(\vec{R}_{D}, t_{i}\right)$ is also independent of $R$. This implies that after $N$ iterations (for arbitrary $N) \gamma_{q q}(t)$ has no $R$ dependence for $0 \leqslant t<R / c$ and hence the causal nature of $P_{\mathrm{III}}(t)$.

\section{CALCULATION OF THE PROBABILITIES UP TO FOURTH ORDER}

For an examination of the fundamental causal properties of the probabilities, it is sufficient to employ a two-level model for each atom. We denote the frequency difference $\left(E_{p}^{S}-E_{g}^{S}\right) / \hbar$ by $\omega^{S}$ and $\left(E_{q}^{D}-E_{g}^{D}\right) / \hbar$ by $\omega^{D}$. The generalization to multilevel atoms is straightforward and does not 
change the conclusions concerning causality reached in this section.

\section{A. Case I}

For this case we calculate the probability of finding the system in a pure state where detector $D$ is excited, the source $S$ has decayed to its ground state, and the field is the vacuum. Starting from the initial state $\mid g, p$; vac $\rangle$, the probability of finding the system in state $\mid q, g$; vac $\rangle$ at time $t$ is, from Eq. (3.12),

$$
P_{\mathrm{I}}(t)=\left|\left\langle\operatorname{vac} ; \overline{0}\left|\beta_{g}^{S}(t) \beta_{q}^{D}(t)\right| g, p ; \mathrm{vac}\right\rangle\right|^{2} .
$$

This probability is shown to be noncausal. Although nonzero for $t<R / c$, it is small except for times $t$ close to $R / c$ and $\omega^{S} \approx \omega^{D}$. For $t>R / c$ and $\omega^{S} \approx \omega^{D}$, the probability is dominated by its causal part. In the general case the leading contribution to the probability amplitude is second order in the dipole transition moments. Using Eq. (3.20), with its second term denoted by $\beta_{n}^{(s)}(t)$, the amplitude may be written as

$$
\begin{aligned}
M(t)= & \left\langle\mathrm{vac} ; \overline{0 \mid}\left[\beta_{g}^{S}(0)+\beta_{g}^{S(s)}(t)\right]\right. \\
& \times\left[\beta_{q}^{D}(0)+\beta_{q}^{D(s)}(t)\right]|g, p ; \mathrm{vac}\rangle \\
= & \left\langle\mathrm{vac} ; \overline{0}\left|\beta_{g}^{S}(0) \beta_{q}^{D(s)}(t)\right| g, p ; \mathrm{vac}\right\rangle \\
& +\left\langle\operatorname{vac} ; \overline{0}\left|\beta_{g}^{S(s)}(t) \beta_{q}^{D(s)}(t)\right| g, p ; \mathrm{vac}\right\rangle \\
= & M_{c}(t)+M_{\mathrm{nc}}(t),
\end{aligned}
$$

where we have used $\beta_{q}(0)|g\rangle=0$. This partitioning corresponds to causal and noncausal terms. Inserting the explicit expression for $\beta_{q}^{D(s)}(t)$, the first term of Eq. (5.2) becomes

$$
\begin{aligned}
M_{c}(t)=\langle & \operatorname{vac} ; \overline{0} \mid \beta_{g}^{S}(0)\left(\frac{i}{\hbar}\right) \mu_{i}(D) \int_{0}^{t} d t^{\prime} \beta_{g}^{D}\left(t^{\prime}\right) \\
& \times d_{i}\left(\vec{R}_{D}, t^{\prime}\right) e^{i \omega^{D} t^{\prime}}|g, p ; \mathrm{vac}\rangle,
\end{aligned}
$$

from which we find the lead term that depends on the product of the two transition moments $\mu(S) \mu(D)$. Since Eq.
(5.3) has $\mu_{i}(D)$ as an explicit factor, $\beta_{g}^{D}\left(t^{\prime}\right)$ can be replaced by $\beta_{g}^{D}(0)$; also it is sufficient to find $\vec{d}\left(\vec{R}_{D}, t^{\prime}\right)$ up to first order in $\mu(S)$. Thus this approximation to $M_{c}(t)$ leads to

$$
\frac{i}{\hbar} \mu_{i}(D) \int_{0}^{t} d t^{\prime} e^{i \omega^{D} t^{\prime}}\left\langle g\left|d_{i}^{S(1)}\left(\vec{R}_{D}, t^{\prime}\right)\right| p\right\rangle,
$$

where the matrix element in Eq. (5.4) is in the source-atom space alone. Since $d_{i}^{S(1)}\left(\vec{R}_{D}, t^{\prime}\right)$ is zero for $t^{\prime}<R / c$, the amplitude (5.4) vanishes for $t<R / c$. For $t^{\prime}>R / c$, we have, from Eq. (3.28),

$$
\begin{aligned}
d_{i}^{S(1)}\left(\vec{r}, t^{\prime}\right)= & \sum_{m, n} \mu_{j}^{m n}(S)\left(-\nabla^{2} \delta_{i j}+\nabla_{i} \nabla_{j}\right) \\
& \times \frac{e^{-i \omega^{S}\left(t^{\prime}-\left|\vec{r}-\vec{R}_{S}\right| c\right)}}{\left|\vec{r}-\vec{R}_{S}\right|} \beta_{m}^{S \dagger}(0) \beta_{n}^{S}(0) .
\end{aligned}
$$

Hence, for $t>R / c$,

$$
\begin{aligned}
M_{c}(t)= & \frac{i}{\hbar} \mu_{i}(D) \mu_{j}(S) \int_{R / c}^{t} d t^{\prime} e^{i \omega^{D} t^{\prime}} \\
& \times\left(-\nabla^{2} \delta_{i j}+\nabla_{i} \nabla_{j}\right) \frac{1}{R} e^{-i \omega^{S}\left(t^{\prime}-R / c\right)} \\
= & \frac{1}{\hbar} \mu_{i}(S) \mu_{j}(D) e^{i\left(\omega^{D}-\omega^{S}\right) R / c} \\
& \times\left[\left(-\nabla^{2} \delta_{i j}+\nabla_{i} \nabla_{j}\right) \frac{c^{i \omega^{S} R / c}}{R}\right] \\
& \times\left[\frac{e^{i\left(\omega^{D}-\omega^{S}\right)(t-R / c)}-1}{\omega^{D}-\omega^{S}}\right]
\end{aligned}
$$

In the large- $R$ limit, the probability arising from Eq. (5.6) is proportional to the inverse square of the separation. We note that the time-dependent factor in Eq. (5.6) is the familiar expression that appears in first-order time-dependent perturbation theory. For near resonance $\omega^{S} \approx \omega^{D}$, the modulus squared leads to Fermi's result [1].

The second term $M_{\mathrm{nc}}(t)$ of the amplitude (5.2) is now evaluated. This is found to depend on the correlation function between the vacuum fluctuations at $S$ and $D$. In contrast to $M_{c}(t)$, this is noncausal. Substituting for the sourcedependent $\beta$ operators in $M_{\mathrm{nc}}(t)$, we get

$$
\begin{aligned}
M_{\mathrm{nc}}(t) & =-\frac{1}{\hbar^{2}} \mu_{i}(D) \mu_{j}(S) \int_{0}^{t} d t^{\prime} e^{-i \omega^{S} t^{\prime}} \int_{0}^{t} d t^{\prime \prime} e^{i \omega^{D} t^{\prime \prime}}\left\langle\operatorname{vac}\left|d_{j}^{(0)}\left(\vec{R}_{S}, t^{\prime}\right) d_{i}^{(0)}\left(\vec{R}_{D}, t^{\prime \prime}\right)\right| \mathrm{vac}\right\rangle \\
& =-\left(\frac{1}{\hbar}\right) \mu_{i}(D) \mu_{j}(S)\left(-\nabla^{2} \delta_{i j}+\nabla_{i} \nabla_{j}\right) \frac{F\left(R, t ; \omega^{S}, \omega^{D}\right)}{R},
\end{aligned}
$$

where $F\left(R, t ; \omega^{S}, \omega^{D}\right)$ is defined by

$$
F\left(R, t ; \omega^{S}, \omega^{D}\right)=\frac{c}{\pi} \int_{0}^{\infty} d k(\sin k R) \frac{\left[e^{i\left(\omega^{D}-\omega^{S}\right) t}-e^{i\left(\omega+\omega^{D}\right) t}-e^{-i\left(\omega+\omega^{S}\right) t}+1\right]}{\left(\omega+\omega^{D}\right)\left(\omega+\omega^{S}\right)} .
$$


The $k$ integral can be expressed in terms of sine and cosine integrals [10]; it does not vanish for $t<R / c$ and hence $M_{\mathrm{nc}}(t)$ is noncausal.

Before discussing the probabilities we consider the above amplitudes in the far zone, namely, $R>\lambda$, where $\lambda$ is the reduced wavelength of a typical atomic transition. In particular we examine the behavior of the noncausal amplitude in the interval $(R-\lambda) / c<t<(R+\lambda) / c$. We make two approximations: first, the far-zone approximation, which results in

$$
M_{\mathrm{nc}}(t) \approx \frac{1}{\hbar} \mu_{i}(D) \mu_{j}(S)\left(\delta_{i j}-\hat{R}_{i} \hat{R}_{j}\right) \frac{1}{R} \frac{d^{2} F}{d R^{2}}
$$

and second, the stationary phase approximation near the point $|c t-R| \approx 0$, which gives

$$
\begin{aligned}
M_{\mathrm{nc}}(t) \approx & \frac{1}{2 \pi \hbar c} \mu_{i}(D) \mu_{j}(S)\left(\delta_{i j}-\hat{R}_{i} \hat{R}_{j}\right) \frac{1}{R(R-c t)} \\
& \times\left(e^{i \omega^{D} t}+e^{-i \omega^{S} t}\right) .
\end{aligned}
$$

This noncausal amplitude is clearly large in the neighborhood $R \approx c t[7(\mathrm{~b})]$. We now compare this with the causal amplitude (5.6). For $R>\lambda$,

$$
\begin{aligned}
& M_{c}(t) \approx \frac{1}{\hbar c^{2}} \mu_{i}(D) \mu_{j}(S)\left(\delta_{i j}-\hat{R}_{i} \hat{R}_{j}\right) \frac{1}{R}\left[\frac{\omega^{S^{2}}}{\omega^{D}-\omega^{S}}\right] \\
& \times\left[e^{i \omega^{S}(R / c-t)}-e^{i \omega^{D}(R / c-t)}\right] e^{i \omega^{D} t}, \quad t>R / c \\
&=0, \quad t<R / c .
\end{aligned}
$$

In the neighborhood of $R \approx c t, M_{c}(t)$ is small; in fact, it is identically zero for $R>c t$. For $R<c t$,

$$
\left|\frac{M_{c}(t)}{M_{\mathrm{nc}}(t)}\right| \approx \frac{(c t-R)^{2}}{\lambda^{2}}
$$

so that the noncausal contribution to the probability $P_{\mathrm{I}}(t)$ is the dominant one for $0<t<(R+\lambda) / c$.

The time-dependent probability $P_{\mathrm{I}}(t)$ in terms of the two amplitudes is

$$
\begin{aligned}
P_{\mathrm{I}}(t)= & \left|M_{c}(t)+M_{\mathrm{nc}}(t)\right|^{2}=\left|M_{c}(t)\right|^{2}+\left[M_{c}(t) \bar{M}_{\mathrm{nc}}(t)\right. \\
& \left.+\bar{M}_{c}(t) M_{\mathrm{nc}}(t)\right]+\left|M_{\mathrm{nc}}(t)\right|^{2} .
\end{aligned}
$$

For $t<R / c$,

$$
\begin{aligned}
P_{\mathrm{I}}(t)=\mid & \left.M_{\mathrm{nc}}(t)\right|^{2}=\frac{1}{\hbar^{2}} \mu_{i}(D) \mu_{j}(S) \mu_{k}(D) \mu_{l}(S) \\
& \times\left[\left(-\nabla^{2} \delta_{i j}+\nabla_{i} \nabla_{j}\right) \frac{F\left(R, t ; \omega^{D}, \omega^{S}\right)}{R}\right] \\
& \times\left[\left(-\nabla^{2} \delta_{k l}+\nabla_{k} \nabla_{l}\right) \frac{\bar{F}\left(R, t ; \omega^{D}, \omega^{S}\right)}{R}\right] .
\end{aligned}
$$

As noted above, this is small except for $R / c>t>(R$ $-\lambda) / c$. In this interval, $P_{\mathrm{I}}(t)$ for a randomly oriented pair is found to be

$$
\begin{aligned}
P_{\mathrm{I}}(t) \approx & \frac{1}{9 \pi^{2} \hbar^{2} c^{2}}|\mu(D)|^{2}|\mu(S)|^{2} \frac{1}{R^{2}(R-c t)^{2}} \\
& \times\left[1+\cos \left(\omega^{D}+\omega^{S}\right) t\right] .
\end{aligned}
$$

For $t>R / c$, the causal term $\left|M_{c}(t)\right|^{2}$ dominates (except in the interval $R / c<t<(R+\lambda) / c$ ) and

$$
\begin{aligned}
P_{\mathrm{I}}(t) \approx & \left|M_{c}(t)\right|^{2}=\frac{1}{\hbar^{2}} \mu_{i}(D) \mu_{j}(S) \mu_{k}(D) \mu_{l}(S)\left[\left(-\nabla^{2} \delta_{i j}\right.\right. \\
& \left.\left.+\nabla_{i} \nabla_{j}\right) \frac{e^{i \omega^{S} R / c}}{R}\right]\left[\left(-\nabla^{2} \delta_{k l}+\nabla_{k} \nabla_{l}\right) \frac{e^{-i \omega^{S} R / c}}{R}\right] \\
& \times\left|\frac{e^{i\left(\omega^{D}-\omega^{S}\right)(t-R / c)}-1}{\left(\omega^{D}-\omega^{S}\right)}\right|^{2}
\end{aligned}
$$

In physically realistic situations, energy transfer between neighboring atoms occurs when $\omega^{D} \approx \omega^{S}$. In such cases, the probability (5.16) dominates because of the near-resonant denominator. In the large- $R$ limit, the probability $(5.16)$ is equivalent to the Fermi result [1].

\section{B. Case II}

For this case, the initial conditions are the same as before, namely, the detector $D$ is in its ground state $S$, the source atom is in the excited state $|p\rangle$, and the radiation field is the vacuum. In contrast to case I, we now find the probability at time $t$ that $D$ is excited and $S$ in its ground state, with the field unspecified. From Eq. (3.15)

$$
P_{\mathrm{II}}(t)=\sum_{F}\left|\left\langle F ; \overline{0 \mid} \beta_{g}^{S}(t) \beta_{q}^{D}(t) \mid g, p ; \mathrm{vac}\right\rangle\right|^{2}
$$

It is clear that up to fourth order in the transition moments, the only field states $|F\rangle$ that can contribute are the vacuum $|v a c\rangle$ and the two-photon states $\left|k, k^{\prime}\right\rangle$. The contribution involving the vacuum is the same as that for case I. So

$$
P_{\mathrm{II}}(t)=P_{\mathrm{I}}(t)+\sum_{\text {modes }}\left|\left\langle k, k^{\prime} ; \overline{0 \mid} \beta_{g}^{S}(t) \beta_{q}^{D}(t) \mid g, p ; \mathrm{vac}\right\rangle\right|^{2}
$$

We now calculate the amplitude up to order $\mu(A) \mu(B)$ for the term involving the two-photon states. This amplitude is

$$
\begin{aligned}
\left\langle k, k^{\prime} ; \overline{0}\right| & \beta_{g}^{S}(t) \beta_{q}^{D}(t)|g, p ; \mathrm{vac}\rangle \\
= & -\frac{1}{\hbar^{2}} \mu_{i}(D) \mu_{j}(S) \int_{0}^{t} d t^{\prime} e^{-i \omega^{S} t^{\prime}} \int_{0}^{t} d t^{\prime \prime} e^{i \omega^{D} t^{\prime \prime}}\left\langle k, k^{\prime}\right| \\
& \times d_{j}^{(0)}\left(\vec{R}_{S}, t^{\prime}\right) d_{i}^{(0)}\left(\vec{R}_{D}, t^{\prime \prime}\right)|\mathrm{vac}\rangle
\end{aligned}
$$

The off-diagonal matrix element in Eq. (5.19) is for the correlation function operator for the vacuum fluctuations at $S$ and $D$ at different times. Hence the amplitude (5.19) is non- 
causal. Using the mode expansion for $\vec{d}^{(0)}$ and performing the time integrals, we obtain for the amplitude

$$
\begin{aligned}
& -\frac{1}{\hbar^{2}} \mu_{i}(D) \mu_{j}(S)\left(\frac{2 \pi \hbar c k}{V}\right)^{1 / 2}\left(\frac{2 \pi \hbar c k^{\prime}}{V}\right)^{1 / 2} \\
& \times\left[\overrightarrow{e_{j}} \overline{e_{i}} e^{-i \vec{k} \cdot \vec{R}_{S}} e^{-i \vec{k} \cdot \vec{R}_{D}} \frac{e^{i\left(\omega^{\prime}-\omega^{S}\right) t}-1}{\omega^{\prime}-\omega^{S}} \frac{e^{i\left(\omega+\omega^{D}\right) t}-1}{\omega+\omega^{D}}\right. \\
& \left.+k \leftrightarrow k^{\prime}\right] .
\end{aligned}
$$

The $R$-dependent terms in the probability for the two-photon final state is found, in terms of the function $F$ defined in Eq. (5.8), to be

$$
\begin{aligned}
& \frac{1}{\hbar^{2}} \mu_{i}(D) \mu_{j}(S) \mu_{k}(D) \mu_{l}(S) \\
& \times\left[\left(-\nabla^{2} \delta_{i j}+\nabla_{i} \nabla_{j}\right) \frac{F\left(R, t ;-\omega^{S}, \omega^{D}\right)}{R}\right] \\
& \times\left[\left(-\nabla^{2} \delta_{k l}+\nabla_{k} \nabla_{l}\right) \frac{\bar{F}\left(R, t ;-\omega^{S}, \omega^{D}\right)}{R}\right] .
\end{aligned}
$$

The noncausal part of $P_{\mathrm{II}}(t)$ is the sum of Eqs. (5.14) and (5.21). Both these terms are small except when $R$ is close to $c t$. Then $P_{\mathrm{II}}(t)$ for a randomly oriented pair is

$$
\begin{aligned}
P_{\mathrm{II}}(t) \approx & \frac{2}{9 \pi^{2} \hbar^{2} c}|\mu(D)|^{2}|\mu(S)|^{2} \frac{1}{R^{2}(R-c t)^{2}} \\
& \times\left[1+\cos \omega^{D} t \cos \omega^{S} t\right] .
\end{aligned}
$$

For $R>c t$, the causal part dominates and is the same as that for case I, namely, Eq. (5.16).

\section{Case III}

For this case, again with the same initial state, we calculate the probability at time $t$ that the detector is excited with no reference to the states of the emitting atom and the radiation field. This probability is causal and an explicit expression for it could be found up to fourth order in the moments using the approach discussed in Sec. IV. However, in this subsection we follow a procedure similar to the one used for cases I and II. This enables us to identify the contributions that cancel the noncausal terms found in the previous cases. The probability $P_{\mathrm{III}}(t)$ may be expressed as

$$
P_{\mathrm{III}}(t)=\sum_{F} \sum_{p^{\prime}}\left|\left\langle F ; \overline{0}\left|\beta_{p^{\prime}}^{S}(t) \beta_{q}^{D}(t)\right| g, p ; \mathrm{vac}\right\rangle\right|^{2},
$$

where the sums are over complete sets of states for the radiation field and for atom $S$, Thus $P_{\mathrm{III}}(t)$ up to fourth order in the moments and in the two-level approximation is

$$
\begin{aligned}
P_{\mathrm{III}}(t)= & \left|\left\langle\mathrm{vac} ; \overline{0 \mid} \beta_{g}^{S}(t) \beta_{q}^{D}(t) \mid g, p ; \mathrm{vac}\right\rangle\right|^{2} \\
& +\sum_{\text {modes }}\left|\left\langle k, k^{\prime} ; \overline{0 \mid} \beta_{g}^{S}(t) \beta_{q}^{D}(t) \mid g, p ; \mathrm{vac}\right\rangle\right|^{2} \\
& +\sum_{\text {modes }}\left|\left\langle k ; \overline{0 \mid} \beta_{p}^{S}(t) \beta_{q}^{D}(t) \mid g, p ; \mathrm{vac}\right\rangle\right|^{2} \\
= & P_{\mathrm{II}}(t)+\sum_{\text {modes }}\left|\left\langle k ; \overline{0 \mid} \beta_{p}^{S}(t) \beta_{q}^{D}(t) \mid g, p ; \mathrm{vac}\right\rangle\right|^{2} .
\end{aligned}
$$

Using the decomposition (3.20), the amplitude of the additional term in Eq. (5.24) can be written as the sum of two matrix elements

$$
\begin{aligned}
& \left\langle k ; \overline{0 \mid} \beta_{p}^{S}(0) \beta_{q}^{D(s)}(t) \mid g, p ; \mathrm{vac}\right\rangle+\langle k ; \overline{0}| \beta_{p}^{S(s)}(t) \\
& \quad \times \beta_{q}^{D(s)}(t)|g, p ; \mathrm{vac}\rangle
\end{aligned}
$$

The first term has a nonvanishing first-order contribution dependent on $\mu(D)$. Hence, to find the probability correct to fourth order in the moments, it is necessary to evaluate the amplitude to third order, namely, to order $\mu(D) \mu^{2}(S)$. The first-order term is immediate and is given by

$$
\begin{aligned}
M^{(1)}(t) & =(i / \hbar) \mu_{i}(D) \int_{0}^{t} d t^{\prime} e^{i \omega^{D} t^{\prime}}\left\langle k\left|d_{i}^{(0)}\left(\vec{R}_{D}, t^{\prime}\right)\right| \mathrm{vac}\right\rangle \\
& =\mu_{i}(D)\left(\frac{2 \pi \hbar c k}{V}\right)^{1 / 2} \overline{e_{i}} e^{-i \vec{k} \cdot \vec{R}_{D}} \frac{e^{i\left(\omega+\omega^{D}\right) t}-1}{\omega+\omega^{D}}
\end{aligned}
$$

There are no terms of order $\mu(D) \mu(S)$. The required thirdorder term arises from both matrix elements of Eq. (5.25). It is

$$
\begin{aligned}
M^{(3)}= & \left\langle k ; \overline{0}\left|\beta_{p}^{S}(0)(t) \beta_{q}^{D(3)}(t)\right| g, p ; \mathrm{vac}\right\rangle+\langle k ; \overline{0}| \beta_{p}^{S(2)}(t) \\
& \times \beta_{q}^{D(1)}(t)|g, p ; \mathrm{vac}\rangle=M_{c}^{(3)}+M_{\mathrm{nc}}^{(3)}
\end{aligned}
$$

As will be seen below, the contribution to the probability from the interference between $M^{(1)}$ and $M_{c}^{3}$ is causal and between $M^{(1)}$ and $M_{\mathrm{nc}}^{(3)}$ is noncausal.

We first obtain the noncausal contribution to the probability and show that it cancels the noncausal terms in $P_{\mathrm{II}}(t)$. We have

$$
\begin{aligned}
M_{\mathrm{nc}}^{(3)}(t)= & \left\langle k ; \overline{0 \mid} \beta_{p}^{S(2)}(t) \beta_{q}^{D(1)}(t) \mid g, p ; \mathrm{vac}\right\rangle \\
= & -\frac{i}{\hbar^{3}} \mu_{k}(D) \mu_{j}(S) \mu_{l}(S) \\
& \times \int_{0}^{t} d t^{\prime \prime \prime} e^{i \omega^{D} t^{\prime \prime \prime}} \int_{0}^{t} d t^{\prime} e^{i \omega^{S} t^{\prime}} \int_{0}^{t^{\prime}} d t^{\prime \prime} e^{-i \omega^{S} t^{\prime \prime}} \\
& \times\left\langle k\left|d_{l}^{(0)}\left(\vec{R}_{S}, t^{\prime \prime}\right) d_{j}^{(0)}\left(\vec{R}_{S}, t^{\prime}\right) d_{k}^{(0)}\left(\vec{R}_{D}, t^{\prime \prime \prime}\right)\right| \mathrm{vac}\right\rangle
\end{aligned}
$$

where we have used the solution (3.20) for the $\beta^{(s)}(t)$ operators. The noncausal contribution to the probability at time $t$ is 


$$
\begin{aligned}
\bar{M}^{(1)}(t) M_{\mathrm{nc}}^{(3)}(t)+M^{(1)}(t) \bar{M}_{\mathrm{nc}}^{(3)}(t) \\
=-\frac{1}{\hbar^{4}} \mu_{i}(D) \mu_{j}(S) \mu_{k}(D) \mu_{l}(S) \\
\quad \times \int_{0}^{t} d t^{\prime} e^{i \omega^{S} t^{\prime}} \int_{0}^{t^{\prime}} d t^{\prime \prime} e^{-i \omega^{S} t^{\prime \prime}} \int_{0}^{t} d t^{\prime \prime \prime} e^{i \omega^{D} t^{\prime \prime \prime}} \\
\quad \times \int_{0}^{t} d t^{\prime \prime \prime \prime} e^{-i \omega^{D} t^{\prime \prime \prime \prime}}\langle\operatorname{vac}| d_{i}^{(0)}\left(\vec{R}_{D}, t^{\prime \prime \prime \prime}\right) \\
\quad \times d_{l}^{(0)}\left(\vec{R}_{S}, t^{\prime \prime}\right) d_{j}^{(0)}\left(\vec{R}_{S}, t^{\prime}\right) d_{k}^{(0)}\left(\vec{R}_{D}, t^{\prime \prime \prime}\right)|\mathrm{vac}\rangle+\text { c.c. }
\end{aligned}
$$

The evaluation of the fourth-order correlation function is straightforward. On inserting a complete set of field states $|F\rangle$ we have

$$
\begin{aligned}
\sum_{F}\langle\operatorname{vac}| d_{i}^{(0)}\left(\vec{R}_{D}, t^{\prime \prime \prime \prime}\right) d_{l}^{(0)}\left(\vec{R}_{S}, t^{\prime \prime}|F\rangle\langle F| d_{j}^{(0)}\left(\vec{R}_{S}, t^{\prime}\right)\right. \\
\quad \times d_{k}^{(0)}\left(\vec{R}_{D}, t^{\prime \prime \prime}\right)|\operatorname{vac}\rangle .
\end{aligned}
$$

The only field states that can contribute are the vacuum and the two-photon states. When $|F\rangle$ is the vacuum, the corresponding contribution to Eq. (5.29) is found to be the negative of Eq. (5.21); when $|F\rangle$ are two-photon states, the contribution is the negative of Eq. (5.14). Hence these cancel the noncausal terms in $P_{\mathrm{II}}(t)$ and $P_{\mathrm{III}}(t) \equiv 0$ for $t<R / c$.

It remains to find an expression for $P_{\mathrm{III}}(t)$ when $t>R / c$. It has two contributions: the causal part of $P_{\mathrm{I}}(t)$, which is the modulus square of the probability amplitude (5.6), and the causal part of the interference term $\bar{M}^{(1)}(t) M_{c}^{(3)}(t)+M^{(1)}(t) \bar{M}_{c}^{(3)}(t)$. The second of these is found in Appendix B. For the case of near resonance $\omega^{D}$ $\approx \omega^{S}$, the lead term arising from interference is

$$
\begin{aligned}
\frac{1}{9 \pi \hbar^{2}} & |\mu(D)|^{2}|\mu(S)|^{2}\left[\left(-\nabla^{2} \delta_{i j}+\nabla_{i} \nabla_{j}\right) \frac{e^{i \omega^{S} R / c}}{R}\right] \\
& \times \frac{1}{\omega^{D}-\omega^{S}} \int_{0}^{\infty} \frac{d \omega}{\left(\omega^{D}+\omega\right)\left(\omega^{S}+\omega\right)} \\
& \times\left[\left(-\nabla^{2} \delta_{i j}+\nabla_{i} \nabla_{j}\right) \frac{\sin (\omega R / c)}{R}\right]\left(e^{-i\left(\omega^{D}+\omega\right) t}-1\right) \\
& \times\left(e^{i\left(\omega^{D}-\omega^{S}\right) t}-e^{i\left(\omega^{D}-\omega^{S}\right) R_{0} / c}\right)+\text { c.c. },
\end{aligned}
$$

which is proportional to $\left(\omega^{D}-\omega^{S}\right)^{-1}$. On the other hand, the term from the square of the amplitude (5.6) that is proportional to $\left(\omega^{D}-\omega^{S}\right)^{-2}$ dominates $P_{\mathrm{III}}(t)$ for $t>R / c$. For randomly oriented molecules, we find from Eq. (5.16)

$$
\begin{aligned}
P_{\mathrm{III}}(t)= & \frac{1}{9 \hbar^{2}}|\mu(D)|^{2}|\mu(S)|^{2}\left[\left(-\nabla^{2} \delta_{i j}+\nabla \nabla_{j}\right) \frac{e^{i \omega^{S} R / c}}{R}\right] \\
& \times\left[\left(-\nabla^{2} \delta_{i j}+\nabla_{i} \nabla_{j}\right) \frac{e^{-i \omega^{S} R / c}}{R}\right] \\
& \times\left[\frac{\sin ^{2}\left\{\left[\left(\omega^{D}-\omega^{S}\right) / 2\right](t-R / c)\right\}}{\left[\left(\omega^{D}-\omega^{S}\right) / 2\right]^{2}}\right] \\
= & \frac{2}{9 \hbar^{2} R^{6}|\mu(D)|^{2}|\mu(S)|^{2}\left\{3+(k R)^{2}+(k R)^{4}\right\}} \\
& \times\left[\frac{\sin ^{2}\left\{\left[\left(\omega^{D}-\omega^{S}\right) / 2\right](t-R / c)\right\}}{\left[\left(\omega^{D}-\omega^{S}\right) / 2\right]^{2}}\right], \quad t>R / c, \quad(5
\end{aligned}
$$

where $c k=\omega^{S} \approx \omega^{D}$. We reiterate that $P_{\mathrm{III}}(t) \equiv 0$ for $t$ $<R / c$.

\section{SUMMARY}

We have used the Heisenberg picture to calculate the excitation transfer probability between two atoms. Fermi's early calculation was based on a complete specification of the final states of the atoms and the field. He employed an approximation that extended the integrals over frequency to the negative domain. This led to a causal result. However, a calculation without this approximation gives a noncausal probability, which has been confirmed with our method. In the present work we have used perturbation theory correct to fourth order in the transition moments to calculate the probabilities for the outcomes in various experimental setups corresponding to different specifications of the system at the time of measurement. These probabilities show in general noncausal behavior. However, for the case where neither the state of the source nor that of the field is specified, the fourth-order result is causal. This probability is a sum of three types of terms, differing in the number of photons in the intermediate states (5.24). Each of these types has an $R$-dependent part that is nonzero for $t<R / c$. Nevertheless, their sum is zero, thus providing an explicit demonstration of the causal behavior up to fourth order. We also have given a formal proof showing that to all orders in the transition moments, the probability is strictly causal when the measurement is inclusive, i.e., the measurement is solely made on the detector.

\section{ACKNOWLEDGMENTS}

We thank D. P. Craig and F. Persico for stimulating discussions concerning this work.

\section{APPENDIX A}

In this appendix we obtain the energy density of the electromagnetic field in the neighborhood of an atom. In particular, the energy density is found at time $t>0$ given that at $t$ $=0$ the atom is known to be in an excited state that has a dipole-allowed transition to the ground state. As mentioned in the Introduction, the time development of the energy density exhibits causal behavior. In the Heisenberg picture the demonstration of this causality is straightforward as the 
Heisenberg source fields are causal [20]. A demonstration within the Schrödinger picture is less direct. We outline the calculation of the energy density (correct to quadratic terms in the transition moments) within this picture and explicitly show the causal behavior. Given that the initial state $|i\rangle$ is $|p ; \mathrm{vac}\rangle$, the state at time $t$, correct to second order, is

$$
|\Psi(t)\rangle=e^{-i H t / \hbar}|i\rangle=\left|\Psi^{(0)}(t)\right\rangle+\left|\Psi^{(1)}(t)\right\rangle+\left|\Psi^{(2)}(t)\right\rangle,
$$

where

$$
\begin{aligned}
&\left|\Psi^{(0)}(t)\right\rangle=e^{-i \omega_{p} t}|p ; \mathrm{vac}\rangle \\
&\left|\Psi^{(1)}(t)\right\rangle=(-i / \hbar) \sum_{\operatorname{modes}} e^{-i\left(\omega_{g}+\omega\right) t}|g ; \vec{k}\rangle\langle\vec{k} ; g| \\
&-\vec{\mu} \cdot \vec{d}|p ; \mathrm{vac}\rangle \int_{0}^{t} d t^{\prime} e^{i\left(\omega-\omega_{0}\right) t^{\prime}},
\end{aligned}
$$

where $\omega_{0}=\omega_{p}-\omega_{g}$.

The change in the energy density at time $t$ is

$$
\left\langle\Psi^{(1)}(t)\left|\frac{d^{2}(\vec{r})}{8 \pi}\right| \Psi^{(1)}(t)\right\rangle+\left\langle\Psi^{(0)}(t)\left|\frac{d^{2}(\vec{r})}{8 \pi}\right| \Psi^{(2)}(t)\right\rangle+\left\langle\Psi^{(2)}(t)\left|\frac{d^{2}(\vec{r})}{8 \pi}\right| \Psi^{(0)}(t)\right\rangle,
$$

with a similar expression for the change in magnetic energy density. In his calculation, Kikuchi [2] used only the first term of Eq. (A5). For a randomly oriented dipole source this term is

$$
\begin{aligned}
\left\langle\Psi^{(1)}(t)\left|\frac{d^{2}(\vec{r})}{8 \pi}\right| \Psi^{(1)}(t)\right\rangle & =\frac{\mu^{2}}{3} \frac{c^{2}}{4 \pi^{3}}\left|\left(-\nabla^{2} \delta_{i j}+\nabla_{i} \nabla_{j}\right) \frac{1}{r} \int_{0}^{\infty} d k(\sin k r) e^{-i k c t} \int_{0}^{t} d t^{\prime} e^{i\left(k-k_{0}\right) c t^{\prime}}\right|^{2} \\
& =\frac{\mu^{2}}{3} \frac{1}{4 \pi^{3}}\left|\left(-\nabla^{2} \delta_{i j}+\nabla_{i} \nabla_{j}\right) \frac{1}{r} \int_{0}^{\infty} d k(\sin k r) e^{-i k c t} \frac{e^{i\left(k-k_{0}\right) c t}-1}{k-k_{0}}\right|^{2}
\end{aligned}
$$

The far-zone limit of Eq. (A7) is essentially Kikuchi's result [Eq. (20) of Ref. [2]]. The integral in Eq. (A7) does not vanish for $r>c t$ and hence this contribution to the energy density is not causal. However, he was able to obtain causal behavior by extending the limits of the integral to include all negative frequencies. We show that this behavior easily follows from Eq. (A6) by extending the $k$ limits before doing the time integral. Using

$$
\int_{-\infty}^{\infty} d k(\sin k r) e^{-i k c t} e^{i\left(k-k_{0}\right) c t^{\prime}}=-i \pi e^{-i k_{0} c t^{\prime}}\left[\delta\left(r-c\left(t-t^{\prime}\right)\right)-\delta\left(r+c\left(t-t^{\prime}\right)\right)\right]
$$

and noting that $t>t^{\prime}$ in the integrand in Eq. (A6), we have

$$
\left\langle\Psi^{(1)}(t)\left|\frac{d^{2}(\vec{r})}{8 \pi}\right| \Psi^{(1)}(t)\right\rangle=\left\{\begin{array}{l}
\left(\mu^{2} / 12 \pi\right)\left|\left(-\nabla^{2} \delta_{i j}+\nabla_{i} \nabla_{j}\right) \frac{e^{i k_{0} r}}{r}\right|^{2} \text { for } t>r / c \\
0 \quad \text { for } t<r / c .
\end{array}\right.
$$

Kikuchi justified the extension of the limits of the $k$ integral on the basis that the integral is small for negative $k$. However, we point out that the added terms, though small, are noncausal. As shown below, the approximation of extending the limits is not needed in a complete calculation. The inclusion of the second and third terms of Eq. (A5) makes the total energy density expression exactly causal.

We now evaluate the second term in Eq. (A5) using Eqs. (A2) and (A4), 


$$
\begin{aligned}
& \left\langle\Psi^{(0)}(t)\left|\frac{d^{2}(\vec{r})}{8 \pi}\right| \Psi^{(2)}(t)\right\rangle=-\frac{1}{8 \pi \hbar} \sum_{\text {modes }} e^{-i\left(k+k^{\prime}\right) c t}\left\langle\operatorname{vac} ; p\left|\frac{d^{2}(\vec{r})}{8 \pi}\right| p ; \vec{k}, \vec{k}^{\prime}\right\rangle\left\langle\vec{k}^{\prime}, \vec{k} ; p|-\vec{\mu} \cdot \vec{d}| g ; \vec{k}\right\rangle\langle\vec{k} ; g| \\
& -\vec{\mu} \cdot \vec{d}|p ; \operatorname{vac}\rangle \int_{0}^{t} d t^{\prime} e^{i\left(k^{\prime}+k_{0}\right) c t^{\prime}} \int_{0}^{t^{\prime}} d t^{\prime \prime} e^{i\left(k-k_{0}\right) c t^{\prime \prime}} \\
& =-\frac{\mu^{2}}{3} \frac{c^{2}}{4 \pi^{3}} \int_{0}^{\infty} d k \int_{0}^{\infty} d k^{\prime}\left[\left(-\nabla^{2} \delta_{i j}+\nabla_{i} \nabla_{j}\right) \frac{\sin k r}{r}\right] \\
& \times\left[\left(-\nabla^{2} \delta_{i j}+\nabla_{i} \nabla_{j}\right) \frac{\sin k^{\prime} r}{r}\right] e^{-i\left(k+k^{\prime}\right) c t} \int_{0}^{t} d t^{\prime} e^{i\left(k^{\prime}+k_{0}\right) c t^{\prime}} \int_{0}^{t^{\prime}} d t^{\prime \prime} e^{i\left(k-k_{0}\right) c t^{\prime \prime}} \\
& =\frac{\mu^{2}}{3} \frac{1}{4 \pi^{3}}\left(-\nabla^{2} \delta_{i j}+\nabla_{i} \nabla_{j}\right)^{r} \frac{1}{r}\left(-\nabla^{2} \delta_{i j}+\nabla_{i} \nabla_{j}\right)^{\bar{r}} \frac{1}{\bar{r}} \int_{0}^{\infty} d k \int_{0}^{\infty} d k^{\prime} \\
& \times \frac{1}{2}\left(\sin k r \sin k^{\prime} \bar{r}+\sin k \bar{r} \sin k^{\prime} r\right) e^{-i\left(k+k^{\prime}\right) c t}\left\{\frac{e^{i\left(k+k^{\prime}\right) c t}-1}{\left(k-k_{0}\right)\left(k+k^{\prime}\right)}-\frac{e^{i\left(k^{\prime}+k_{0}\right) c t}-1}{\left(k-k_{0}\right)\left(k^{\prime}+k_{0}\right)}\right\},
\end{aligned}
$$

where, for convenience, we have introduced $\bar{r}$ (to be set equal to $r$ after the differentiations). The third term in Eq. (A5) is the complex conjugate of Eq. (A12). Hence the change in the total energy density (A5) can be written as

$$
\begin{aligned}
\frac{\mu^{2}}{3} \frac{1}{4 \pi^{3}}( & \left.-\nabla^{2} \delta_{i j}+\nabla_{i} \nabla_{j}\right)^{r} \frac{1}{r}\left(-\nabla^{2} \delta_{i j}+\nabla_{i} \nabla_{j}\right) \bar{r} \times \frac{1}{\bar{r}}\left\{\int_{0}^{\infty} d k \int_{0}^{\infty} d k^{\prime} \frac{1}{2}\left(\sin k r \sin k^{\prime} \bar{r}+\sin k \bar{r} \sin k^{\prime} r\right)\right. \\
& \times\left[\frac{1-e^{-i\left(k^{\prime}-k_{0}\right) c t}-e^{i\left(k-k_{0}\right) c t}+e^{i\left(k-k^{\prime}\right) c t}}{\left(k-k_{0}\right)\left(k^{\prime}-k_{0}\right)}+\frac{1-e^{-i\left(k+k^{\prime}\right) c t}}{\left(k+k^{\prime}\right)\left(k-k_{0}\right)}-\frac{e^{-i\left(k-k_{0}\right) c t}-e^{-i\left(k+k^{\prime}\right) c t}}{\left(k^{\prime}+k_{0}\right)\left(k-k_{0}\right)}+\frac{1-e^{i\left(k+k^{\prime}\right) c t}}{\left(k+k^{\prime}\right)\left(k-k_{0}\right)}\right. \\
& \left.\left.-\frac{e^{i\left(k-k_{0}\right) c t}-e^{i\left(k+k^{\prime}\right) c t}}{\left(k^{\prime}+k_{0}\right)\left(k-k_{0}\right)}\right]\right\} .
\end{aligned}
$$

We consider separately the integrals within the curly brackets with different numerators: For terms with no time dependence in the numerator

$$
\begin{aligned}
\frac{1}{2} \int_{0}^{\infty} & d k \int_{0}^{\infty} d k^{\prime}\left(\sin k r \sin k^{\prime} \bar{r}+\sin k \bar{r} \sin k^{\prime} r\right) \\
& \times\left[\frac{1}{\left(k-k_{0}\right)\left(k^{\prime}-k_{0}\right)}+\frac{2}{\left(k+k^{\prime}\right)\left(k-k_{0}\right)}\right] \\
= & \frac{1}{2} \int_{0}^{\infty} d k \int_{0}^{\infty} d k^{\prime}\left(\sin k r \sin k^{\prime} \bar{r}+\sin k \bar{r} \sin k^{\prime} r\right) \\
& \times\left[\frac{1}{\left(k^{\prime}-k\right)\left(k-k_{0}\right)}-\frac{1}{\left(k^{\prime}-k\right)\left(k^{\prime}-k_{0}\right)}\right. \\
& \left.+\frac{2}{\left(k+k^{\prime}\right)\left(k-k_{0}\right)}\right]=\int_{0}^{\infty} \frac{d k \int_{0}^{\infty} d k^{\prime}\left(\sin k r \sin k^{\prime} \bar{r}\right.}{} \\
& \left.+\sin k \bar{r} \sin k^{\prime} r\right) \frac{1}{k-k_{0}}\left(\frac{1}{\left(k+k^{\prime}\right)}+\frac{1}{\left(k^{\prime}-k\right)}\right)(\mathrm{A} 1
\end{aligned}
$$

the $k^{\prime}$ integral is elementary and Eq. (A14) becomes

$$
\pi \int_{0}^{\infty} d k \frac{\sin k(r+\bar{r})}{k-k_{0}}
$$

and for terms with $e^{ \pm i\left(k-k^{\prime}\right) c t}$ and $e^{ \pm i\left(k+k^{\prime}\right) c t}$

$$
\begin{aligned}
\frac{1}{2} \int_{0}^{\infty} & d k \int_{0}^{\infty} d k^{\prime}\left(\sin k r \sin k^{\prime} \bar{r}+\sin k \bar{r} \sin k^{\prime} r\right) \\
& \times\left[\frac{e^{i\left(k-k^{\prime}\right) c t}}{k-k^{\prime}}\left(\frac{1}{k^{\prime}-k_{0}}-\frac{1}{k-k_{0}}\right)\right. \\
& \left.+\frac{e^{-i\left(k-k^{\prime}\right) c t}}{k+k^{\prime}}\left(\frac{1}{k^{\prime}+k_{0}}\right)+\frac{e^{i\left(k-k^{\prime}\right) c t}}{k+k^{\prime}}\left(\frac{1}{k^{\prime}+k_{0}}\right)\right] \\
= & \frac{1}{2} \int_{0}^{\infty} d k \int_{-\infty}^{\infty} d k^{\prime} \sin k r \sin k^{\prime} \bar{r} \\
& \times\left[\frac{e^{i\left(k-k^{\prime}\right) c t}}{\left(k-k^{\prime}\right)\left(k^{\prime}-k_{0}\right)}-\text { c.c. }\right]+r \leftrightarrow \bar{r}
\end{aligned}
$$

the $k^{\prime}$ integral depends on the sign of $r-c t$. For $r>c t$, Eq. (A16) becomes

$$
\pi \int_{0}^{\infty} d k \frac{\sin k r}{k-k_{0}}\left[-\cos k \bar{r}+\cos \left(k-k_{0}\right) c t \cos k_{0} \bar{r}\right]+r \leftrightarrow \bar{r}
$$

For $r<c t$, Eq. (A16) becomes 


$$
\pi \int_{0}^{\infty} d k \frac{\sin k r}{k-k_{0}} \sin \left[\left(k-k_{0}\right) c t\right] \sin k_{0} \bar{r}+r \leftrightarrow \bar{r} .
$$

For terms with $e^{-i\left(k^{\prime}-k_{0}\right) c t}$ and $e^{i\left(k-k_{0}\right) c t}$

$$
\begin{aligned}
& {\left[-\frac{1}{2} \int_{0}^{\infty} d k \int_{0}^{\infty} d k^{\prime} \sin k r \frac{\sin k^{\prime} \bar{r}}{k^{\prime}-k_{0}} e^{-i\left(k^{\prime}-k_{0}\right) c t}\right.} \\
& \left.\times\left(\frac{1}{\left(k-k_{0}\right)}+\frac{1}{\left(k+k_{0}\right)}\right)+\text { c.c. }\right]+r \leftrightarrow \bar{r} .
\end{aligned}
$$

After interchanging $k$ and $k^{\prime}$, the $k^{\prime}$ integral in Eq. (A19) becomes

$$
\begin{aligned}
& {\left[-\frac{1}{2} \int_{-\infty}^{\infty} d k^{\prime} \frac{\sin k^{\prime} \bar{r}}{k^{\prime}-k_{0}} \int_{0}^{\infty} d k \frac{\sin k r}{k-k_{0}} e^{-i\left(k-k_{0}\right) c t}+\text { c.c. }\right]} \\
& +r \leftrightarrow \bar{r}=-\pi \int_{0}^{\infty} d k \frac{\sin k r}{k-k_{0}} \cos \left[\left(k-k_{0}\right) c t\right] \cos k_{0} \bar{r} \\
& +r \leftrightarrow \bar{r},
\end{aligned}
$$

independent of the sign of $r-c t$.

We now show that the total energy density from the three contributions vanishes for $t<r / c$, which demonstrates strict causal behavior. Of the three contributions, Eqs. (A15) and (A20) hold for all $t$, whereas the second contribution (A16) has different forms depending on whether $t<r / c$ or $t>r / c$. For $t<r / c$, the contribution (A17) is

$$
\begin{gathered}
-\pi \int_{0}^{\infty} d k \frac{\sin k(r+\bar{r})}{k-k_{0}}+\pi \int_{0}^{\infty} d k \cos \left(k-k_{0}\right) c t \\
\times \frac{\sin k r \cos k_{0} \bar{r}+\sin k \bar{r} \cos k_{0} r}{k-k_{0}},
\end{gathered}
$$

which is the negative of the sum of Eqs. (A15) and (A20). Thus, for $t<r / c$ the change in energy density is strictly zero.

We conclude this appendix by obtaining an expression for the electric energy density for $t>r / c$. In contrast to the above, the second contribution is now different and is given by Eq. (A18); it does not cancel the other terms. Taking the three contributions (A15), (A18), and (A20) together, we find for the energy density

$$
\begin{aligned}
\left\langle\Psi(t)\left|\frac{d^{2}(\vec{r})}{8 \pi}\right| \Psi(t)\right\rangle= & \frac{\mu^{2}}{12 \pi^{2}}\left(-\nabla^{2} \delta_{i j}+\nabla_{i} \nabla_{j}\right)^{r} \frac{1}{r}\left(-\nabla^{2} \delta_{i j}+\nabla_{i} \nabla_{j}\right)^{\bar{r}} \frac{1}{\bar{r}} \int_{0}^{\infty} d k \frac{\sin k r}{k-k_{0}}\left\{\cos k \bar{r}+\sin \left[\left(k-k_{0}\right) c t\right] \sin k_{0} \bar{r}\right. \\
& \left.-\cos \left[\left(k-k_{0}\right) c t\right] \cos k_{0} \vec{r}\right\}+\left.r \leftrightarrow \bar{r}\right|_{\bar{r}=r} \\
= & \frac{\mu^{2}}{12 \pi^{2}}\left(-\nabla^{2} \delta_{i j}+\nabla_{i} \nabla_{j}\right)^{r} \frac{1}{r}\left(-\nabla^{2} \delta_{i j}+\nabla_{i} \nabla_{j}\right)^{\bar{r}} \overline{\bar{r}} \\
& \times\left\{\int_{-\infty}^{\infty} d k \frac{\sin k r}{k-k_{0}}\left\{\cos k \bar{r}-\cos \left[k c t-k_{0}(c t-r)\right]+r \leftrightarrow \vec{r}\right\}\right. \\
& \left.-\int_{0}^{\infty} d k \frac{\sin k r}{k+k_{0}}\left\{\cos k \bar{r}-\cos \left[k c t+k_{0}(c t-r)\right]+r \leftrightarrow \vec{r}\right\}\right\}\left.\right|_{\bar{r}=r} .
\end{aligned}
$$

The first integral within the large curly brackets in Eq. (A22) is straightforward and is $\pi \cos \left[k_{0}(r-\bar{r})\right]$. So its contribution to the energy density is time independent and is

$$
\begin{aligned}
\frac{\mu^{2}}{12 \pi^{2}} & \left(-\nabla^{2} \delta_{i j}+\nabla_{i} \nabla_{j}\right)^{r} \frac{1}{r} \\
\times & \left(-\nabla^{2} \delta_{i j}+\nabla_{i} \nabla_{j}\right)^{\bar{r}} \frac{1}{\bar{r}} \cos \left[k_{0}(r-\bar{r})\right] \\
= & \frac{\mu^{2}}{12 \pi^{2}}\left|\left(-\nabla^{2} \delta_{i j}+\nabla_{i} \nabla_{j}\right) \frac{e^{i k_{0} r}}{r}\right|^{2},
\end{aligned}
$$

which simplifies to

$$
\frac{\mu^{2} k_{0}^{4}}{6 \pi r^{2}}\left(1+\frac{1}{k_{0}^{2} r^{2}}+\frac{3}{k_{0}^{4} r^{4}}\right)
$$

The second integral in Eq. (A22) has both time-independent and time-dependent contributions. The time-dependent con- tribution can be expressed as an integral over imaginary frequencies as in Eq. (3.26) of Ref. [20]. It vanishes for times larger than the Bohr period. Finally, the time-independent part of the corresponding energy density to be added to Eq. (A24) is that encountered in the Casimir-Polder potential. It is

$$
\begin{aligned}
& -\frac{\mu^{2}}{12 \pi^{2}}\left(-\nabla^{2} \delta_{i j}+\nabla_{i} \nabla_{j}\right)^{r} \frac{1}{r}\left(-\nabla^{2} \delta_{i j}+\nabla_{i} \nabla_{j}\right)^{\bar{r}} \frac{1}{\bar{r}} \\
& \quad \times \int_{0}^{\infty} d k \frac{\sin [k(r+\bar{r})]}{k-k_{0}} \\
& =-\frac{\mu^{2}}{12 \pi^{2}}\left(-\nabla^{2} \delta_{i j}+\nabla_{i} \nabla_{j}\right)^{r} \frac{1}{r} \\
& \quad \times\left.\left(-\nabla^{2} \delta_{i j}+\nabla_{i} \nabla_{j}\right)^{\bar{r}} \frac{1}{\bar{r}} k_{0} \int_{0}^{\infty} d u \frac{e^{-u(r+\bar{r})}}{u^{2}+k_{0}^{2}}\right|_{\bar{r}=r},
\end{aligned}
$$


which after differentiation becomes

$$
\begin{aligned}
-\frac{\mu^{2} k_{0}}{6 \pi^{2}} \int_{0}^{\infty} d u \frac{u^{6} e^{-2 u r}}{u^{2}+k_{0}^{2}} & \\
& \times\left[\frac{1}{u^{2} r^{2}}+\frac{2}{u^{3} r^{3}}+\frac{5}{u^{4} r^{4}}+\frac{6}{u^{5} r^{5}}+\frac{3}{u^{6} r^{6}}\right] .
\end{aligned}
$$

For large $r$, Eq. (A26) falls off with the power law $r^{-7}$, whereas Eq. (A24) shows an inverse square law dependence that arises from real photon emission. It is interesting to note that for an atom in its ground state, the electric energy density is solely given by Eq. (A26), with $k_{0}$ taking a negative sign.

\section{APPENDIX B}

In this appendix we obtain the causal part of the interference term that contributes to the probability for case III. It is

$$
\bar{M}^{(1)}(t) M_{c}^{(3)}(t)+M^{(1)}(t) \bar{M}_{c}^{(3)}(t),
$$

where

$$
M^{(1)}(t)=\left\langle k ; \overline{0}\left|\beta_{p}^{S}(0) \beta_{q}^{D(1)}(t)\right| g, p ; \mathrm{vac}\right\rangle
$$

and

$$
M_{c}^{(3)}(t)=\left\langle k ; \overline{0}\left|\beta_{p}^{S}(0) \beta_{q}^{D(3)}(t)\right| g, p ; \mathrm{vac}\right\rangle .
$$

From Eq. (3.20)

$$
\beta_{q}^{D(1)}(t)=\frac{i}{\hbar} \mu_{i}(D) \int_{0}^{t} d t^{\prime} e^{i \omega^{D} t^{\prime}} \beta_{g}^{D}(0) d_{i}^{(0)}\left(\vec{R}_{D}, t^{\prime}\right)
$$

Hence

$$
M^{(1)}(t)=\frac{i}{\hbar} \mu_{i}(D) \int_{0}^{t} d t^{\prime} e^{i \omega^{D} t^{\prime}}\left\langle k\left|d_{i}^{(0)}\left(\vec{R}_{D}, t^{\prime}\right)\right| \mathrm{vac}\right\rangle .
$$

Again, from Eq. (3.20)

$$
\beta_{q}^{D(3)}(t)=\frac{i}{\hbar} \mu_{i}(D) \int_{0}^{t} d t^{\prime} e^{i \omega^{D} t^{\prime}} \beta_{g}^{D}(0) d_{i}^{(2)}\left(\vec{R}_{D}, t^{\prime}\right),
$$

where we have ignored terms that would contribute to order $|\mu(D)|^{3}$ to the probability. Hence

$$
M_{c}^{(3)}(t)=\frac{i}{\hbar} \mu_{i}(D) \int_{0}^{t} d t^{\prime} e^{i \omega^{D} t^{\prime}}\left\langle k\left|d_{i}^{(2)}\left(\vec{R}_{D}, t^{\prime}\right)\right| \mathrm{vac}\right\rangle .
$$

The interference term (B1) is

$$
\begin{aligned}
& \frac{1}{\hbar^{2}} \mu_{i}(D) \mu_{j}(D) \int_{0}^{t} d t^{\prime \prime \prime} e^{-i \omega^{D} t^{\prime \prime \prime}} \int_{0}^{t} d t^{\prime} e^{i \omega^{D} t^{\prime}} \\
& \quad \times\left\langle\operatorname{vac}\left|d_{i}^{(0)}\left(\vec{R}_{D}, t^{\prime \prime \prime}\right)\right| k\right\rangle\left\langle k\left|d_{j}^{(2)}\left(\vec{R}_{D}, t^{\prime}\right)\right| \mathrm{vac}\right\rangle+\text { c.c. }
\end{aligned}
$$

The Maxwell field operator $d_{j}^{(2)}\left(\vec{R}_{D}, t^{\prime}\right)$ that is needed in Eq. (B8) arises from the source $S$. This is clearly causal. For $t^{\prime}>$ $R / c$, it is found from Eq. (3.28) to be

$$
\begin{aligned}
\left\langle p\left|d_{j}^{S(2)}\left(\vec{R}_{D}, t^{\prime}\right)\right| p\right\rangle= & \mu_{k}(B)\left(-\nabla^{2} \delta_{j k}+\nabla_{j} \nabla_{k}\right) \frac{1}{R} \\
& \times\left[\left\langlep\left|\beta_{g}^{\dagger(1)}\left(t^{\prime}-R / c\right)\right| \overline{0} e^{-i \omega^{S}\left(t^{\prime}-R / c\right)}\right.\right. \\
& \left.+\left\langle\overline{0} \beta_{g}^{(1)}\left(t^{\prime}-R / c\right) \mid p\right\rangle e^{i \omega^{S}\left(t^{\prime}-R / c\right)}\right] .
\end{aligned}
$$

Substituting Eq. (B9) into Eq. (B8) and using

$$
\begin{aligned}
\left\langle\overline{0 \mid} \beta_{g}^{(1)}\left(t^{\prime}-R / c\right) \mid p\right\rangle= & \frac{i}{\hbar} \mu_{l}(S) \int_{0}^{t^{\prime}-R / c} d t^{\prime \prime} e^{-i \omega^{S} t^{\prime \prime}} \\
& \times d_{l}^{(0)}\left(\vec{R}_{S}, t^{\prime \prime}\right),
\end{aligned}
$$

we get for the interference term

$$
\begin{gathered}
\frac{i}{\hbar^{3}} \mu_{i}(D) \mu_{j}(D) \mu_{k}(S) \mu_{l}(S) \int_{0}^{t} d t^{\prime \prime \prime} e^{-i \omega^{D} t^{\prime \prime \prime}} \int_{R / c}^{t} d t^{\prime} e^{i \omega^{D} t^{\prime}}\left(-\nabla^{2} \delta_{j k}+\nabla_{j} \nabla_{k}\right) \frac{1}{R} \int_{0}^{t^{\prime}-R / c} d t^{\prime \prime}\left\langle\operatorname{vac}\left|d_{i}^{(0)}\left(\vec{R}_{D}, t^{\prime \prime \prime}\right)\right| k\right\rangle \\
\times\left\langle k\left|d_{l}^{(0)}\left(\vec{R}_{S}, t^{\prime \prime}\right)\right| \operatorname{vac}\right\rangle\left[e^{-i \omega^{S} t^{\prime \prime}} e^{i \omega^{S}\left(t^{\prime}-R / c\right)}-e^{i \omega^{S} t^{\prime \prime}} e^{-i \omega^{S}\left(t^{\prime}-R / c\right)}\right]+\text { c.c. } \\
=\frac{i}{\pi \hbar^{2}} \mu_{i}(D) \mu_{j}(D) \mu_{k}(S) \mu_{l}(S)\left(-\nabla^{2} \delta_{j k}+\nabla_{j} \nabla_{k}\right)^{R} \frac{1}{R}\left(-\nabla^{2} \delta_{i l}+\nabla_{i} \nabla_{l}\right)^{\frac{1}{R}} \frac{1}{\bar{R}} \int_{0}^{\infty} d \omega \sin (\omega \bar{R} / c) \\
\quad \times \int_{0}^{t} d t^{\prime \prime \prime \prime} e^{-i\left(\omega^{D}+\omega\right) t^{\prime \prime \prime}} \int_{R_{0} / c}^{t} d t^{\prime} \int_{0}^{t^{\prime}-R / c} d t^{\prime \prime}\left[e^{-i \omega^{S} R / c} e^{i\left(\omega^{D}+\omega^{S}\right) t^{\prime}} e^{-i\left(\omega^{S}-\omega\right) t^{\prime \prime}}-e^{i \omega^{S} R / c} e^{i\left(\omega^{D}-\omega^{S}\right) t^{\prime}} e^{\left.i\left(\omega^{S}+\omega\right) t^{\prime \prime}\right]+\mathrm{c} . c .}\right.
\end{gathered}
$$

After performing the time integrals, we find the interference term to be 


$$
\begin{aligned}
& \bar{M}^{(1)}(t) M_{c}^{(3)}(t)+M^{(1)}(t) \bar{M}_{c}^{(3)}(t)=-\frac{1}{\pi \hbar^{2}} \mu_{i}(D) \mu_{j}(D) \mu_{k}(S) \mu_{l}(S)\left(-\nabla^{2} \delta_{j k}+\nabla_{j} \nabla_{k}\right)^{R} \frac{1}{R}\left(-\nabla^{2} \delta_{i l}+\nabla_{i} \nabla_{l}\right)^{R} \frac{1}{\bar{R}} \\
& \times \int_{0}^{\infty} d \omega \sin (\omega \bar{R} / c) \frac{e^{-i\left(\omega^{D}+\omega\right) t}-1}{\omega^{D}+\omega}\left[e^{-i \omega R / c} \frac{e^{i\left(\omega^{D}+\omega\right) t}-e^{i\left(\omega^{D}+\omega\right) R_{0} / c}}{\left(\omega^{S}+\omega\right)\left(\omega^{D}+\omega\right)}\right. \\
&\left.-e^{i \omega^{S} R / c} \frac{e^{i\left(\omega^{D}-\omega^{S}\right) t}-e^{i\left(\omega^{D}-\omega^{S}\right) R_{0} / c}}{\left(\omega^{S}+\omega\right)\left(\omega^{D}-\omega^{S}\right)}-\left(\text { terms with } \omega^{S} \text { changed to }-\omega^{S}\right)\right]+ \text { c.c., } \\
& t>R / c .
\end{aligned}
$$

Expressions (B11)-(B13) are evaluated, after differentiations with respect to $R$ and $\bar{R}$, by setting $R=\bar{R}=R_{0}$.

[1] E. Fermi, Rev. Mod. Phys. 4, 87 (1932).

[2] S. Kikuchi, Z. Phys. 66, 558 (1930).

[3] J. Hamilton, Proc. Phys. Soc. London, Sect. A 62, 12 (1949).

[4] P. W. Milonni and P. L. Knight, Phys. Rev. A 10, 1096 (1974).

[5] E. A. Power and T. Thirunamachandran, Phys. Rev. A 28, 2671 (1983).

[6] W. H. Louisell, Quantum Statistical Properties of Radiation (Wiley, New York, 1973), p. 314.

[7] (a) M. I. Shirokov, Yad. Fiz. 4, 1077 (1966) [Sov. J. Nucl. Phys. 4, 774 (1967)]; (b) Usp. Fiz. Nauk 124, 697 (1978) [Sov. Phys. Usp. 21, 345 (1978)].

[8] A. Valentini, Phys. Lett. A 153, 321 (1991).

[9] M. H. Rubin, Phys. Rev. D 35, 3836 (1987).

[10] D. P. Craig and T. Thirunamachandran, Chem. Phys. 167, 229 (1992).

[11] B. Ferretti, in Old and New Problems in Elementary Particles, edited by G. Puppi (Academic, New York, 1968), p. 108.

[12] A. K. Biswas, G. Compagno, R. Passante, and F. Persico, Phys. Rev. A 42, 4291 (1990); G. Compagno, G. M. Palma, R. Passante, and F. Persico, Chem. Phys. 198, 19 (1995).

[13] P. W. Milonni, D. F. V. James, and H. Fearn, Phys. Rev. A 52, 1525 (1995).

[14] M. de Haan, Physica A 132, 375 (1985).

[15] D. J. Kaup and V. I. Rupasov, J. Phys. A 29, 2149 (1996).

[16] G. C. Hegerfeldt, Phys. Rev. Lett. 72, 596 (1994).

[17] D. Buchloz and J. Yngvason, Phys. Rev. Lett. 73, 613 (1994).

[18] D. P. Craig and T. Thirunamachandran, Chem. Phys. 135, 37 (1989).

[19] E. A. Power and T. Thirunamachandran, Phys. Rev. A 28, 2663 (1983).

[20] E. A. Power and T. Thirunamachandran, Phys. Rev. A 45, 54 (1992). 